\title{
Controllable excitation of gap plasmons by electron beams in metallic nanowire pairs
}

\author{
Wei Cai, ${ }^{1, *}$ Lei Wang, ${ }^{1}$ Xinzheng Zhang, ${ }^{1}$ Jingjun $\mathrm{Xu},{ }^{1}$ and F. Javier García de Abajo ${ }^{2}$ \\ ${ }^{1}$ The Key Laboratory of Weak-Light Nonlinear Photonics, Ministry of Education, School of Physics and TEDA Applied Physics School, \\ Nankai University, Tianjin 300457, China \\ ${ }^{2}$ Instituto de Óptica, CSIC, Serrano 121, 28006 Madrid, Spain
}

(Received 9 June 2010; revised manuscript received 9 August 2010; published 29 September 2010)

\begin{abstract}
We show that electron beams can efficiently excite gap-plasmon modes in metallic waveguides defined by nanowire pairs. These modes are characterized by monopole-monopole charge symmetry. Significant excitation yields of higher-order dipole-dipole and quadrupole-quadrupole modes are also predicted. Our results are based on rigorous numerical solution of Maxwell's equations in which the electron is described as a classical external current. In our analysis, we find it convenient to simulate electron energy-loss spectra, which are resolved in the frequency and parallel momentum transferred from the electron to the wires for various perpendicular electron trajectories. The charge symmetry of these modes is inferred from the distribution of the induced near fields. The emission yield for the gap modes is as high as $10^{-4}$ plasmons per incoming electron over the visible range of frequencies. Higher-order modes can be selectively excited by playing with the orientation of the electron trajectory whereas the gap plasmon is preferentially launched in nonsymmetric configurations and it is totally suppressed in symmetric arrangements. The effect of curvature along the direction of the wires is also explored by considering the excitation of plasmons defined in the gap between two circularly shaped wires. Our results provide full support for the excitation of gap plasmons with designated electron-beam sources in nanocircuits.
\end{abstract}

DOI: $10.1103 /$ PhysRevB.82.125454

PACS number(s): 73.20.Mf, 79.20.Uv, 78.60.Hk, 78.20.Bh

\section{INTRODUCTION}

Surface plasmons are quantized collective oscillations of conduction electrons that can propagate along metallic surfaces. ${ }^{1,2}$ Their potential application for on-chip communication and signal processing has stimulated recent investigations of different geometries for plasmon waveguiding intended to optimize the compromise between propagation distance, degree of confinement, and electric field enhancement. Among these structures, interesting results have been reported for particle chains, ${ }^{3,4}$ grooves, ${ }^{5}$ and wedges ${ }^{6}$ carved in planar surfaces, gaps between metallic nanowires, ${ }^{7}$ and metallodielectric structures. ${ }^{8}$ In particular, gap plasmons have been shown to be excellent candidates to minimize crosstalk between neighboring waveguides, thus allowing a high degree of spatial integration.

However, the excitation of propagating plasmons at designated positions remains a challenge, and this is where fast electrons can make a difference. Recent studies have experimentally demonstrated that swift electrons can produce plasmons with nanometer control over the position of the electron beam. ${ }^{9,10}$ The yield of plasmon generation can reach high levels in different types of waveguides, including particle chains, planar surfaces, and metallic nanowires. ${ }^{11}$ In a related context, localized plasmons have also been excited by electron beams, giving rise to characteristic cathodoluminescence emission features that allow one to measure their spatial variations with the characteristic nanometer resolution of electron microscopes. ${ }^{12-17}$

Propagating gap plasmons are well understood from a theoretical point of view. ${ }^{7}$ Notice that these plasmons are quite different from the resonances that one obtains associated to the excitation of wire pairs using light incident normal to the wires. In particular, retardation in the response of the system is required to have a realistic description of trapped propagating modes. However, our emphasis here is not on the characteristics of these modes but rather on the mechanism and efficiency of their excitation by fast electron beams.

In this paper, we discuss the generation of propagating gap plasmons in silver nanowire pairs by electron-beam excitation. The plasmon excitation probability is calculated by rigorously solving Maxwell's equations using the boundary element method (BEM) ${ }^{18}$ as explained in Sec. II, with the electrons described as classical external point charge-current distributions. We analyze the excited plasmon modes and quantitatively assess the excitation yields in Sec. III. Some results for curved waveguides are also discussed. Our main conclusion is that electron bombardment provides a realistic way of generating gap plasmons with high efficiency at spatially controlled locations, as discussed in Sec. IV, where we analyze the practicality of using fast electrons for plasmon generation.

\section{CALCULATION METHOD}

Fast electrons can launch plasmons in laterally confined waveguides, for instance, in a metallic wire ${ }^{14}$ or in any translationally invariant structure. As a result of this, a detectable electron energy-loss signal should be produced. It is useful to consider the full energy-loss signal, that is, the probability that the electron transfers energy to the medium, which defines the electron energy-loss spectrum (EELS) given by

$$
\Gamma(\omega)=\int_{0}^{\infty} d k_{\|} \Gamma\left(k_{\|}, \omega\right) .
$$

Notice that we have decomposed this quantity here into contributions arising from different energy losses $\hbar \omega$ and wave- 

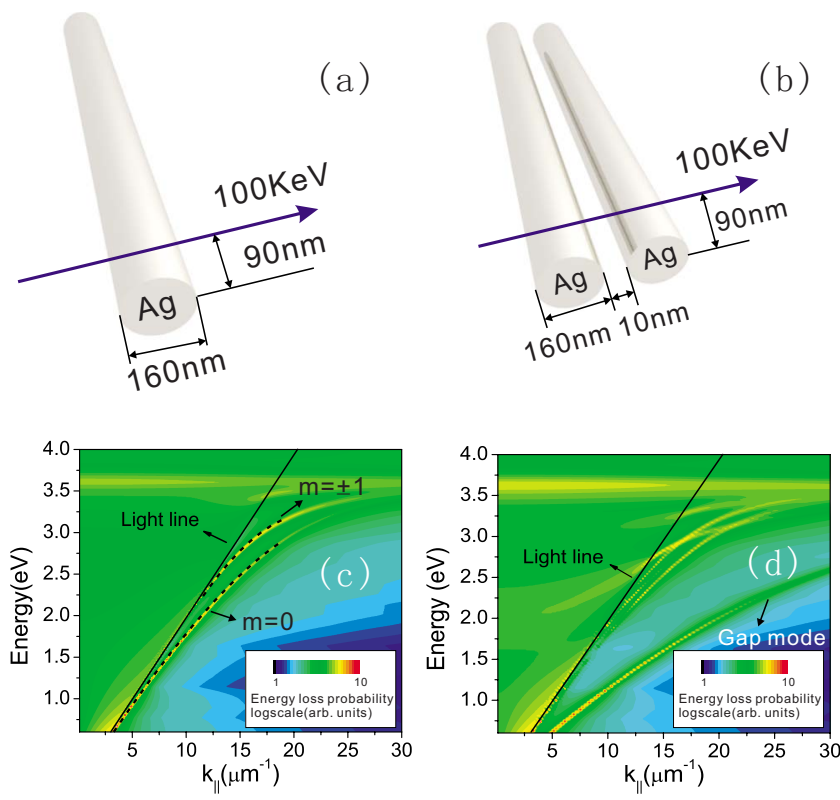

FIG. 1. (Color online) Coupling of an electron beam to metallicwire plasmons. (a) and (b) show schematic views of electrons passing at a distance of $10 \mathrm{~nm}$ from the surface of an infinitely long free-standing silver wire of $160 \mathrm{~nm}$ in diameter and an infinitely long silver wire pair, respectively. (c) Electron energy-loss probability for the single wire considered in (a). The probability is decomposed into contributions arising from different values of the wavevector transfer along the wire axis $k_{\|}$, according to Eq. (1). The probability exhibits clear maxima corresponding to excitation of plasmon modes with $m=0$ and $m= \pm 1$ azimuthal symmetry. (d) Energy-loss probability for the wire pair of (b). The gap-plasmon mode is observed as the lowest-energy feature for each value of $k_{\|}$, and five other higher-order plasmon modes are also resolved.

vector components $k_{\|}$parallel to the direction of translational symmetry (the wires in the present case). All fields, and, in particular, those associated to plasmon modes, can be naturally decomposed in $\left(k_{\|}, \omega\right)$ components, corresponding to a time-space dependence given by $\exp \left(i k_{\|} x-i \omega t\right)$, where the direction $x$ is taken parallel to the wires (see Ref. 7 for a detailed study of the gap modes within this decomposition).

The probability $\Gamma$ is obtained from the retarding force produced by the induced electric field acting back on the electron as ${ }^{19,20}$

$$
\Gamma\left(k_{\|}, \omega\right)=\frac{e}{\pi^{2} \hbar \omega} \int d t \operatorname{Re}\left\{e^{-i \omega t} \mathbf{v} \cdot \mathbf{E}^{\text {ind }}\left(k_{\|}, b, v t, \omega\right)\right\},
$$

where

$$
\mathbf{E}^{\text {ind }}\left(k_{\|}, b, v t, \omega\right)=\int d x e^{-i k_{\|} x} \mathbf{E}^{\text {ind }}(x, b, v t, \omega)
$$

is the Fourier transform of the induced field along $x$, the electron trajectory is described by $z=v t$ (we take $z$ perpendicular to the wires), and $b$ is the so-called impact parameter of the electron beam relative to the wire axis (see Fig. 1).

The quantity $\Gamma\left(k_{\|}, \omega\right)$ is directly related to the photonic local density of states in the vicinity of the wires, ${ }^{21}$ which we calculate from the solution of Maxwell's equations for a translationally invariant geometry using BEM. ${ }^{18,20}$ In Sec. III, we present numerical results of $\Gamma\left(k_{\|}, \omega\right)$ for systems formed by one or two nanowires and electron trajectories perpendicular to the wire axes. Finally, we are also reporting calculations for curved wires in which we have employed a different version of the BEM, taking advantage of rotational symmetry rather than translational invariance. ${ }^{18}$

\section{EXCITATION OF GAP PLASMONS BY ELECTRON BEAMS}

Before looking into the excitation of gap plasmons, let us examine the basic features of plasmon launching in a single nanowire, as shown in Figs. 1(a)-1(c). We investigate the plasmon generation probability for an electron passing near an infinite silver wire of circular cross section and $160 \mathrm{~nm}$ in diameter. We take the dielectric function of silver from tabulated optical data. ${ }^{22}$ Figure $1(\mathrm{c})$ shows that the electron is efficiently exciting plasmons with $m=0$ symmetry [ $m$ enters through the decomposition of the fields into different components with $\exp (\operatorname{im} \phi)$ dependence on azimuthal angle $\phi]$. Also, $m= \pm 1$ modes are excited with lower probability at high energies as compared to the $m=0$ mode. Further details of these modes and their $m$ assignment are found in the literature. ${ }^{7}$ In particular, the dispersion relation of the modes in an infinite cylinder including retardation admits a closedform expression that explicitly depends on $m$ and that we have used to corroborate the $m$ assignment specified in the figure. $^{23}$

Next, we consider a wire pair formed by two $160 \mathrm{~nm}$ circular silver wires with a gap distance of $10 \mathrm{~nm}$, as shown in Fig. 1(b). The contour plot of Fig. 1(d) shows the EELS probability resolved in contributions coming from different wave vectors $k_{\|}$along the direction of the wires. In contrast to the single wire, the electron beam can now excite more plasmon modes, and, in particular, a strongly bound mode shows up with $k_{\|}$well above $\omega / c$, the wave vector of light in free space. This is the so-called gap plasmon, which is basically confined to the gap region between the wires. This mode has been recently shown to provide a reasonable basis for achieving record levels of integration in plasmon circuits. $^{7}$

In order to further explore the symmetry of the different modes excited by the electron in a wire pair, we zoom out their corresponding dispersion diagram, a shown in Fig. 2(a). Apart from the gap-plasmon mode (labeled A), five other bound modes are clearly discernible outside the light cone. We label them B-F. For each of these plasmon modes, we select a single point $\left(k_{\|}, \omega\right)$ along its dispersion relation and calculate the induced near-field distribution within the transversal plane. The orientations of the resulting electric field distributions for modes $\mathrm{A}-\mathrm{F}$ are schematically shown in Fig. 2(b). A localized maximum intensity is observed in the gap region of the wire pair associated to modes $A, C$, and $F$ while minimum intensity is found for modes B and D. The spatial extension of the gap mode $\mathrm{A}$ is limited to the interwire region. This mode is actually made of two-dimensional monopoles in the transverse plane [notice that a monopole in a wire is possible in a two-dimensional configuration because 

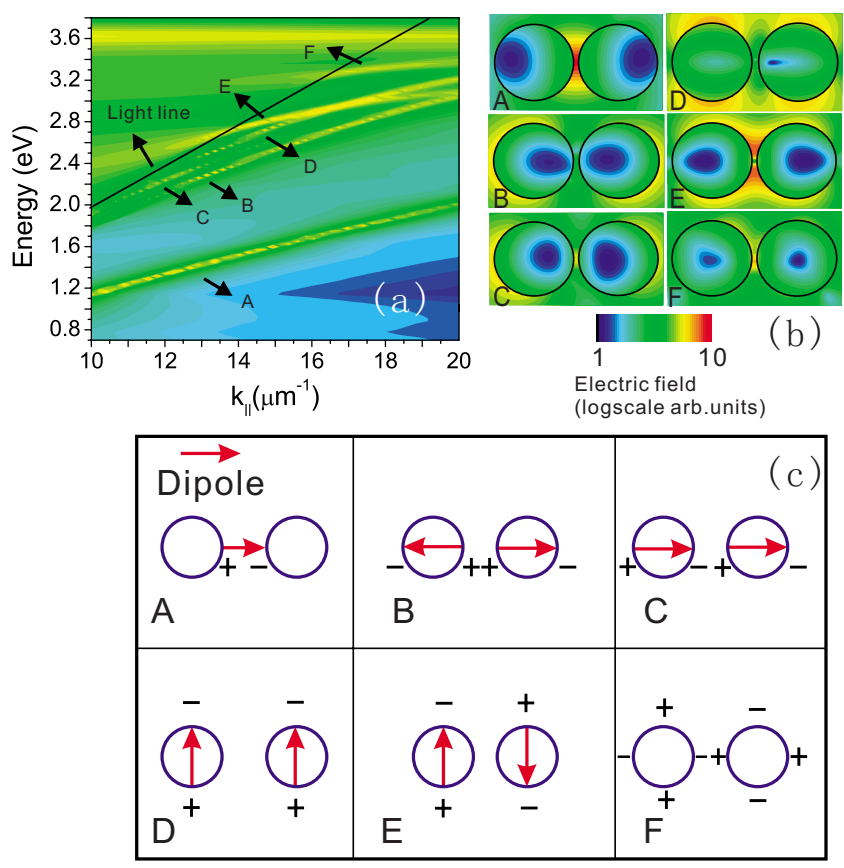

FIG. 2. (Color online) Symmetry and charge ordering of the modes excited by electron beams in the nanowire pair of Fig. 1(b). (a) Enlargement of Fig. 1(d) with the dispersion relation of the plasmon modes excited by an electron beam. The gap plasmon mode is labeled A, and other higher-order modes are labeled B-F. (b) Distribution of the induced electric field intensity for modes A-F (see labels), as calculated for $\left(k_{\|}, \hbar \omega\right)=\left(14.5 \mu \mathrm{m}^{-1}, 1.58 \mathrm{eV}\right), \quad\left(14.5 \mu \mathrm{m}^{-1}\right.$, $2.48 \mathrm{eV}), \quad\left(14.5 \mu \mathrm{m}^{-1}, 2.57 \mathrm{eV}\right), \quad\left(14.5 \mu \mathrm{m}^{-1}, 2.66 \mathrm{eV}\right)$, $\left(14.5 \mu \mathrm{m}^{-1}, 2.79 \mathrm{eV}\right)$, and $\left(18.0 \mu \mathrm{m}^{-1}, 3.30 \mathrm{eV}\right)$, respectively. (c) Surface charge ordering of a nanowire pair for modes A-F. The gap mode $\mathrm{A}$ is a monopole-monopole excitation whereas modes B-E are coupled dipole-dipole modes with different orientation and symmetry, and mode $\mathrm{F}$ is a coupled-quadrupoles mode.

charge neutrality can still be satisfied due to the oscillatory behavior $\exp \left(i k_{\|} x\right)$ along the remaining third dimension $x$, parallel to the wires]. In contrast, upon inspection of the various electric field components (not shown), we find that modes B-E are coupled dipole-dipole plasmons with different orientations and symmetries, as shown in Fig. 2(c). Modes $\mathrm{B}$ and $\mathrm{D}$ are symmetrical whereas modes $\mathrm{C}$ and $\mathrm{E}$ are antisymmetrical with respect to the pair center. The electron can also excite nondipolar modes, which would be difficult to resolve using external light instead. This is the case of mode F, which is a coupled-quadrupoles plasmon. In brief, a gap plasmon mode, four coupled-dipoles modes, and one coupled-quadrupoles mode can be resolved in the dispersion diagram obtained by electron-beam excitation in the wire pair under consideration. Incidently, modes $\mathrm{D}$ and $\mathrm{E}$ are crossing each other at $\left(k_{\|} \hbar \omega\right)=\left(17.0 \mu \mathrm{m}^{-1}, 3.0 \mathrm{eV}\right)$, and this could be potentially useful for the generation of entangled plasmons, with application to quantum information technology.

We consider two other excitation geometries, as shown in Fig. 3(a). Figures 3(b) and 3(c) show dispersion relations of excited plasmons under the conditions of II and III, respectively, and these results must be compared with Fig. 1(d) for
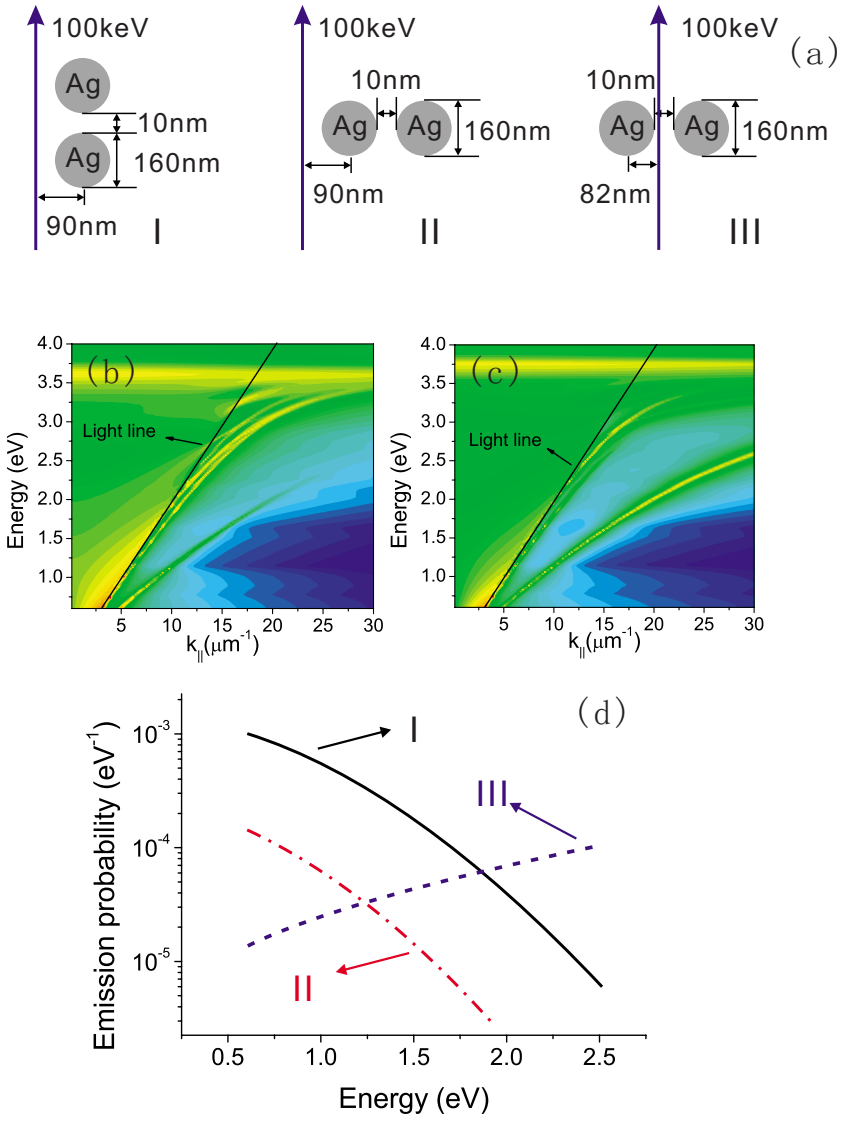

FIG. 3. (Color online) Gap-plasmon excitation probability for different orientations of the electron trajectory relative to a wire pair. (a) Schematic representation of three different electrontrajectory configurations (I, II, and III), all of them perpendicular to the wire axes. (b) and (c) Electron energy-loss probability for configurations II and III, respectively. The probability for I is given in Fig. 1(d). The coupled-dipoles modes B and C and the coupledquadrupoles mode $\mathrm{F}$ are generated with larger probability in II while the gap-plasmon mode $\mathrm{A}$ and mode $\mathrm{C}$ are more intense in III. (d) EELS probability integrated over parallel momentum for the gap mode of configurations I (solid curve), II (dashed-dotted curve), and III (dashed curve).

configuration I. We observe that the situations considered in II and III produce similar results to those found for I: the electron beam is also exciting six plasmon modes. However, due to the different location of the electron trajectory, the efficiency in the excitation of those modes is also different. For configuration I, the electron beam is more productive in the excitation of modes D, E, and F. In contrast, for configuration II the plasmon modes $\mathrm{B}, \mathrm{C}$, and $\mathrm{F}$ are dominating over the remaining modes. Finally, for configuration III the gap mode and modes $\mathrm{C}$ and $\mathrm{F}$ exhibit higher intensity. This is consistent with the requirement that the electric field in the gap (i.e., the region through which the electron is passing) should be a localized maximum. Additionally, higher-order modes can be optimally excited by changing the excitation geometry.

We next turn to discussing the overall probability for exciting gap plasmons. The yield is obtained from the integral of $\Gamma\left(k_{\|} \omega\right)$ over $k_{\|}$in the region near the gap-mode dispersion 

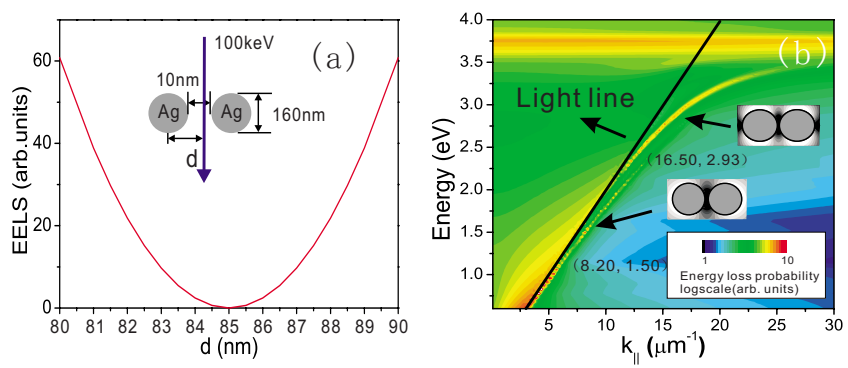

FIG. 4. (Color online) (a) Excitation probability of gap plasmons produced by electrons passing through the gap between a nanowires pair as a function of the distance from the trajectory to the center of the leftmost wire $d$. (b) Dispersion diagram of excited plasmon modes for $d=85 \mathrm{~nm}$ (symmetric configuration) in which the gap plasmon is not present. The insets show the near-field distribution of the plasmon modes that are excited in this case (B and D): $\left(k_{\|}, \hbar \omega\right)=\left(8.20 \mu \mathrm{m}^{-1}, 1.50 \mathrm{eV}\right)$ and $\left(16.50 \mu \mathrm{m}^{-1}, 2.93 \mathrm{eV}\right)$, respectively.

curve. The results are shown in Fig. 3(d) for the three trajectories under consideration as a function of plasmon frequency $\omega$. The emission probability for each of them is on the order of $10^{-4}$ plasmons per incoming electron over the visible and near-infrared range of plasmon frequencies. Furthermore, the variation in the excitation yield with plasmon energy in configurations I and II has different trend as compared to configuration III. Examining Fig. 3(d), we conclude that scheme I is the optimum choice for gap plasmon excitation in the red and near-infrared parts of the spectrum.

We analyze in Fig. 4 the variation in the excitation probability under a configuration similar to III, as a function of electron-beam position across the gap between the nanowires. As expected, the probability vanishes when the electrons pass through the center of the system because the gap mode is antisymmetric [see scheme A in Fig. 2(c)]. In that case, only higher-order, symmetric modes can couple to the electron, as shown in Fig. 4(b). The gap-mode excitation probability increases when the electron moves further away from the center of the structure, and it actually reaches values above those observed for configurations I and II at frequencies in the blue part of the spectrum, as shown in Fig. 3.

Finally, we explore in Fig. 5 the excitation of gap plasmons in a curved-waveguide configuration. An electron is passing near the gap formed by two neighboring waveguides patterned into a ringlike shape, as shown in Fig. 5(a). The resulting EELS probability [Fig. 5(b)] shows distinct sharp peaks that correspond to the excitation of different standing waves formed by the gap plasmon confined within the closed-circuit configuration of this system. The standingwave character of these excitations is clearly resolved in Fig. 5(c), where we show the electric near-field strength at the gap as a function of the angle along the ring. The intensity of these standing waves depends on the azimuthal angle $\phi$ as $\cos ^{2}(n \phi)$. The peak labels in Fig. 5(b) show the corresponding values of $n$ in the 1-7 range. Incidentally, the standing waves display a maximum at $\phi=0$, which coincides with the position of closest approach from the electron trajectory. The inverse time-Fourier transform allows us to go from the frequency-space representation of Fig. 5(c) to the real-space
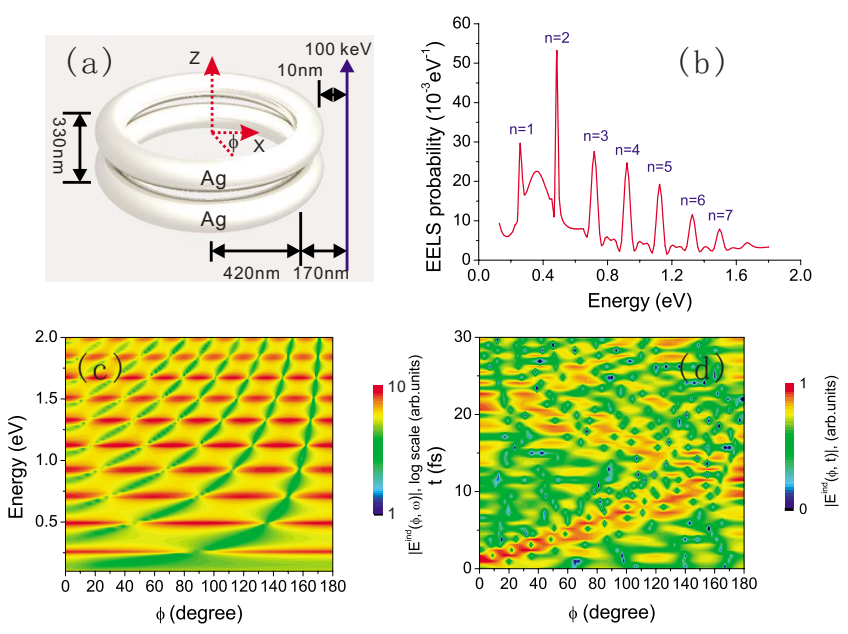

FIG. 5. (Color online) Excitation of gap plasmons in a curvedwaveguide geometry. (a) Curved waveguide formed by two neighboring nanowires patterned into a ringlike shape. (b) EELS produced by a beam as indicated in (a). (c) Variation in the electric near-field strength at the center of the gap as a function of the angle $\phi$ along the ring. The beam is passing close to the $\phi=0$ position. The electric field is resolved in different photon energy components. (d) Time evolution of the field strength along the gap. The electron is closest to the gap at $t=0$.

representation of Fig. 5(d), where one can clearly observe how the electron launches a gap-plasmon wave packet that evolves along the gap with a speed $\sim 0.43 c$, which is very close to the value of the group velocity all along the $0.2-3.0$ $\mathrm{eV}$ energy window [see Fig. 1(d)] over which the gap plasmon is efficiently excited [see Fig. 5(b)].

\section{CONCLUSIONS}

We have shown that fast electrons passing near a pair of parallel silver nanowires can efficiently generate propagating surface plasmons, and, in particular, the plasmons that are confined within the gap region between the wires (gap plasmons). An electron can thus be regarded as a localized source of plasmon excitations. Specifically, we have shown that under the conditions considered in Figs. 2-4, it can create one gap plasmon, four coupled-dipoles modes, and one coupled-quadrupoles mode for each value of the wave vector along the direction parallel to the wires. We find that gap modes are preferentially excited when the electron is passing outside and close to both wires, and, in particular, we obtain a yield as high as $10^{-4}$ plasmons per electron within the visible range of frequencies. Higher-order modes can selectively be excited by changing the orientation of the trajectory relative to the wire pair.

Our study suggests an efficient method to create gapplasmon modes in nanowire pairs, the experimental verification of which should be feasible by examining the cathodoluminescence signal measured in electron microscopes. Since the gap-plasmon propagation distance is several tens of microns, ${ }^{7}$ there is room to separate the position of electron impact from the end of the wires; the latter can act as an outcoupler in which the plasmons are partially transformed 
into emitted light, to be recorded by an optical setup. This optical signal should exhibit oscillations as a function of the distance from the electron-beam position to the end of the waveguide, thus providing access to the dispersion relation of the guided modes. ${ }^{24}$ In another possible arrangement, the wire pairs can be shaped into circuits giving rise to standing waves (e.g., the modes circulating around a circularly patterned waveguide such as that of Fig. 5), and therefore, producing emission features that are controlled by the geometry of the circuit shape.

In a practical arrangement, for eventual application to nanoplasmonic devices, one would need to have an electron beam that is passing at a designated position near the wire pair. Electron microscopes offer this possibility but they are extremely complicated machines that allow one to raster the beam near a sample and focusing it with subnanometer resolution. In contrast, for plasmon generation, the beam cross section can be several nanometers in size, which is one order of magnitude less demanding than what electron microscopes generally offer. Besides, there are other less costly alternatives for producing an electron beam aimed at a fixed position. For example, one can think of an electron gun which produces a beam that is later collimated at a distance of several microns from the wires using a couple of metal blinders. Alternatively, one could use a field emission tip placed close to the wires. At this point, it is convenient to keep in mind that the excitation of guided modes with light is still far from efficient, specially when the modes are well confined, such as in the case of gap plasmons. In this context, the electrons are advantageous in that they can directly excite plasmons that are trapped to the waveguide; this is a direct result of the fact that the electrons can provide the necessary momentum exceeding that of light in the vacuum surrounding the wires. In contrast, a complex optical setup would be required to excite guided plasmons using light (e.g., in the Otto configuration).

\section{ACKNOWLEDGMENTS}

This work has been supported by the Fundamental Research Funds for the Central Universities, the National Natural Science Foundation of China (Grant No. 10874093), the National Basic Research Program of China (Grants No. 2007CB307002 and No. 2010CB934101), the 111 Project (B07013), and the Spanish MICINN (Grant No. MAT200766050 and Consolider NanoLight.es).
*Corresponding author: weicai@nankai.edu.cn

${ }^{1}$ W. L. Barnes, A. Dereux, and T. W. Ebbesen, Nature (London) 424, 824 (2003).

${ }^{2}$ R. Zia, J. A. Schuller, A. Chandran, and M. L. Brongersma, Mater. Today 9, 20 (2006).

${ }^{3}$ M. Quinten, A. Leitner, J. R. Krenn, and F. R. Aussenegg, Opt. Lett. 23, 1331 (1998).

${ }^{4}$ S. A. Maier, P. G. Kik, H. A. Atwater, S. Meltzer, E. Harel, B. E. Koel, and A. A. G. Requicha, Nature Mater. 2, 229 (2003).

${ }^{5}$ S. I. Bozhevolnyi, V. S. Volkov, E. Devaux, J. Y. Laluet, and T. W. Ebbesen, Nature (London) 440, 508 (2006).

${ }^{6}$ E. Moreno, S. G. Rodrigo, S. I. Bozhevolnyi, L. Martín-Moreno, and F. J. García-Vidal, Phys. Rev. Lett. 100, 023901 (2008).

${ }^{7}$ A. Manjavacas and F. J. García de Abajo, Nano Lett. 9, 1285 (2009).

${ }^{8}$ R. F. Oulton, V. J. Sorger, D. A. Genov, D. F. P. Pile, and X. Zhang, Nat. Photonics 2, 496 (2008).

${ }^{9}$ M. V. Bashevoy, F. Jonsson, A. V. Krasavin, N. I. Zheludev, Y. Chen, and M. I. Stockman, Nano Lett. 6, 1113 (2006).

${ }^{10}$ J. T. van Wijngaarden, E. Verhagen, A. Polman, C. E. Ross, H. J. Lezec, and H. A. Atwater, Appl. Phys. Lett. 88, 221111 (2006).

${ }^{11}$ W. Cai, R. Sainidou, J. Xu, A. Polman, and F. J. García de Abajo, Nano Lett. 9, 1176 (2009).
${ }^{12}$ N. Yamamoto, K. Araya, and F. J. García de Abajo, Phys. Rev. B 64, 205419 (2001).

${ }^{13}$ N. Yamamoto, M. Nakano, and T. Suzuki, Surf. Interface Anal. 38, 1725 (2006).

${ }^{14}$ E. J. R. Vesseur, R. de Waele, M. Kuttge, and A. Polman, Nano Lett. 7, 2843 (2007).

${ }^{15}$ R. Gómez-Medina, N. Yamamoto, M. Nakano, and F. J. García de Abajo, New J. Phys. 10, 105009 (2008).

${ }^{16}$ M. V. Bashevoy, F. Jonsson, K. F. MacDonald, Y. Chen, and N. I. Zheludev, Opt. Express 15, 11313 (2007).

${ }^{17}$ M. Kuttge, W. Cai, F. J. García de Abajo, and A. Polman, Phys. Rev. B 80, 033409 (2009).

${ }^{18}$ F. J. García de Abajo and A. Howie, Phys. Rev. B 65, 115418 (2002).

${ }^{19}$ R. H. Ritchie, Phys. Rev. 106, 874 (1957).

${ }^{20}$ F. J. García de Abajo, Rev. Mod. Phys. 82, 209 (2010).

${ }^{21}$ F. J. García de Abajo and M. Kociak, Phys. Rev. Lett. 100, 106804 (2008).

${ }^{22}$ P. B. Johnson and R. W. Christy, Phys. Rev. B 6, 4370 (1972).

${ }^{23}$ J. C. Ashley and L. C. Emerson, Surf. Sci. 41, 615 (1974).

${ }^{24}$ M. Kuttge, E. J. R. Vesseur, A. F. Koenderink, H. J. Lezec, H. A. Atwater, F. J. García de Abajo, and A. Polman, Phys. Rev. B 79, 113405 (2009). 